\title{
Silencing of PROS1 induces apoptosis and inhibits migration and invasion of glioblastoma multiforme cells
}

\author{
MOHD FIRDAUS CHE MAT ${ }^{1}$, NOR AZIAN ABDUL MURAD ${ }^{1}$, KAMARIAH IBRAHIM $^{1}$, \\ NORFILZA MOHD MOKHTAR ${ }^{1,2}$, WAN ZURINAH WAN NGAH ${ }^{1,3}$, ROSLAN HARUN ${ }^{1,4}$ and RAHMAN JAMAL ${ }^{1}$ \\ ${ }^{1}$ UKM Medical Molecular Biology Institute, Universiti Kebangsaan Malaysia; \\ Departments of ${ }^{2}$ Physiology and ${ }^{3}$ Biochemistry, Faculty of Medicine, Universiti Kebangsaan Malaysia, \\ Cheras, Kuala Lumpur 56000; ${ }^{4}$ KPJ Ampang Puteri Specialist Hospital, Ampang, Selangor 68000, Malaysia
}

Received July 11, 2016; Accepted September 14, 2016

DOI: $10.3892 /$ ijo.2016.3755

\begin{abstract}
Glioblastoma multiforme (GBM) is an aggressive brain tumor and most patients have poor prognosis. Despite many advances in research, there has been no significant improvement in the patient survival rate. New molecular therapies are being studied and RNA interference (RNAi) therapy is one of the promising approaches to improve prognosis and increase survival in patients with GBM. We performed a meta-analysis of five different microarray datasets and identified 460 significantly upregulated genes in GBM. Loss-of-function screening of these upregulated genes using LN18 cells was performed to identify the significant target genes for glioma. Further investigations were performed using siRNA in LN18 cells and various functional assays were carried out on the selected candidate gene to understand further its role in GBM. We identified PROSI as a candidate gene for GBM from the meta-analysis and RNAi screening. Knockdown of PROS1 in LN18 cells significantly induced apoptosis compared to siPROS1-untreated cells $(p<0.05)$. Migration in cells treated with siPROS1 was reduced significantly $(\mathrm{p}<0.05)$ and this was confirmed with wound-healing assay. PROS1 knockdown showed substantial reduction in cell invasion up to $82 \%(\mathrm{p}<0.01)$. In addition, inhibition of PROS1 leads to decrease in cellular proliferation by $18 \%$. Knockdown of PROSI in LN18 cells caused activation of both of the extrinsic and intrinsic apoptotic pathways. It caused major upregulation of FasL which is important for death receptor signaling activation and also downregulation of GAS6 and other members of TAM family of receptors. PROS1 may play an important role in the development of
\end{abstract}

Correspondence to: Dr Rahman Jamal, UKM Medical Molecular Biology Institute, Universiti Kebangsaan Malaysia, Jalan Yaacob Latif, Bandar Tun Razak, Cheras, Kuala Lumpur 56000, Malaysia E-mail: rahmanj@ppukm.ukm.edu.my

Key words: cancer, glioblastoma, RNA interference screening, PROS1
GBM through cellular proliferation, migration and invasion as well as apoptosis. Targeting PROS1 in GBM could be a novel therapeutic strategy in GBM treatment.

\section{Introduction}

Glioblastoma multiforme (GBM) is the most common primary brain tumor in adults with a median survival rate of only 15 months $(1,2)$. GBM originates from the glial cells which subsequently evolved into tumors known as glioma (3). It is the deadliest primary brain tumor and is classified as grade IV astrocytoma by WHO criteria $(1,4,5)$. The standard treatment for GBM consists of surgical removal, radiation followed by chemotherapy. Unfortunately, these treatments only gave minor improvements to the patients' survival particularly for the recurrent GBM (4).

GBM is resistant towards treatment due to the heterogeneous nature of the disease. These are contributed by the dysregulation of the core signaling pathways such as the $E r b B, M A P K, m T O R$ and $p 53$ signaling pathways (6-9). It was shown that the pathogenesis of GBM requires alteration of multiple genetic pathways and each of the primary and secondary GBMs has a unique combination of these genetic changes (5). Primary or de novo GBM frequently showed loss of heterozygosity (LOH) at $10 \mathrm{q}$ ( $70 \%$ of cases), EGFR amplification (36\%), p16INK4a deletion (31\%) and PTEN mutations (25\%) (10). Mutations in the IDH1 and $I D H 2$ genes are common in astrocytomas, oligodendrogliomas, oligoastrocytomas and secondary glioblastoma with prevalence of 50-80\% of cases (11). In addition, TP53 and PTEN mutations are common in primary and secondary gliomas with a frequency of 28 and $65 \%$ for $p 53,25$ and $4 \%$ for PTEN respectively (10). Secondary GBM is generally initiated from diffuse astrocytomas. Some common molecular lesions associated with secondary GBM are TP53 mutations (60-65\%) and gain of $7 \mathrm{q}$ arm (21-50\%) with $M E T$ gene gain of function (47\% in primary and $44 \%$ in secondary glioblastoma) being affected significantly and associated with poor prognosis (12). There are also reports on the involvement of aberrant intrinsic and extrinsic apoptotic pathways and the overexpression of anti-apoptotic proteins such as FLIPs, BCL2 and survivin which contribute to apoptotic resistance (13-17). 
The therapeutic strategy in GBM could be improved by targeting the multiple pathways involved. RNA interference (RNAi) is one of the attractive approaches and may result in the post-transcriptional knockdown of the genes of interest. Significant impact in in vitro experiments using RNAi has allowed the implementation of therapeutic approach using RNAi gene therapy in vivo (18). One of the major problems in GBM therapy is the difficulty for the drugs to cross the blood-brain barrier (BBB) hindering maximal drug distribution to the tumor site. To date, there are a few strategies being used to efficiently deliver siRNA through the BBB. The RNAi-based nanomedicine platform has been introduced at the pre-clinical stage (18). Based on spherical nucleic acid gold nanoparticle conjugates, which are densely packed, highly oriented siRNA duplexes targeting the oncoprotein Bcl2Like12 (Bcl2L12) were used to neutralize the oncogene expression in GBM (19). There are many delivery systems that form complexes with siRNA including PEGylated immunoliposomes that carry siEGFR, recombinant adeno-associated virus carrying siHec-1 and lentiviral vectors carrying siTRAIL (20-22). An example of a molecular target that has made to clinical trial using siRNA and showed promising results is Tenascin-C (TN-C), which is overexpressed in the extracellular matrix (ECM) of GBM. It has been shown that dsRNA targeting TN-C mRNA could reduce the tumor size significantly and increase the survival rate by $11 \%(23,24)$.

\section{Materials and methods}

Meta-analysis of microarray datasets. We performed a meta-analysis on five microarray datasets from a cancer microarray database using an integrated data-mining platform, the Oncomine Research Edition (25). Data were filtered based on data source, cancer, the type of datasets and analysis. Candidate genes were selected based on the median rank and $\mathrm{p}<0.05$. Candidate genes obtained from meta-analysis were then screened using synthetic lethal RNAi screening and the hits were selected based on their significant values in viability reduction. The human glioblastoma LN18 (TP53-mutant) cells were transfected with pooled siRNA (SMARTpool ${ }^{\mathrm{TM}}$; GE Dharmacon, Lafayette, CO, USA) targeting against 460 genes and cultured for $48 \mathrm{~h}$ according to the manufacturer's protocol. The media were changed after $48 \mathrm{~h}$ post-transfection and incubated for another $48 \mathrm{~h}$. The cells were then prepared for viability measurement using the CellTiter-Glo ${ }^{\circledR}$ Luminescent Cell Viability Assay (Promega Corp., Madison, WI, USA). The experiment was performed in triplicate.

Cell culture. LN18 cells were maintained in T-75 flasks and allowed to grow in $15 \mathrm{ml}$ of Dulbecco's modified Eagle's medium (DMEM) (ATCC, Manassas, VA, USA) and supplemented with $10 \%$ fetal bovine serum (J R Scientific, Inc., Woodland, CA, USA) until $80 \%$ confluency. The cells were incubated under $5 \% \mathrm{CO}_{2}$ condition. Generally, the doubling time for LN18 cells was $<24 \mathrm{~h}$. Cells were harvested by removing media and cells were then washed with $5 \mathrm{ml}$ of $1 \mathrm{X}$ Dulbecco's Phosphate-Buffered Saline (Gibco, Grand Island, NY, USA) and trypsinised using $1 \mathrm{X}$ Trypsin EDTA $0.25 \%$ (J R Scientific, Inc.).
Preparation of siRNA. ON-TARGETplus SMARTpool ${ }^{\mathrm{TM}}$ of PROS1 siRNA (GE Dharmacon) consisting of four different siRNA sequences were used in this experiment. The siRNA sequences used were: i) GCAUGGAAGUGAAUAUUAA; ii) GCAACAGGCUUCACAAGUC; iii) UAUUAGAGC UCACUCAUGU; and iv) GAAGAGUUGUGAGGUUGUU. Lyophilized PROS1 siRNA was resuspended with $1 \mathrm{X}$ siRNA buffer (Thermo Fisher Scientific, Inc., Rockford, IL, USA). A total of $25 \mathrm{nM}$ final concentration of PROS1 siRNA and non-targeting siRNA were used with DharmaFECT2. All functional assays were performed $48 \mathrm{~h}$ post-transfection.

RNA extraction and $q P C R$. RNeasy kit (Qiagen, Hilden, Germany) was used to isolate total RNA from cells. The quality and quantity of the isolated RNA were determined using NanoDrop (Thermo Fisher Scientific, Inc.). Briefly, $100 \mathrm{ng}$ of RNA were used to generate cDNA using iScript $^{\mathrm{TM}}$ cDNA Synthesis kit (Bio-Rad Laboratories, Inc., Hercules, CA, USA). qPCR was conducted using SsoFast ${ }^{\mathrm{TM}}$ EvaGreen ${ }^{\circledR}$ Supermix (Bio-Rad Laboratories, Inc.) on a Rotor-Gene 3000 (Corbett Life Science/Qiagen, Inc., Valencia, CA, USA) platform. The PROS1 primers used were: forward, 5'-TGCTGGCGTGTCTCCTCCTA-3' and reverse, 5'-CAGTTCTTCGATGCATTCTCTTTCA-3'. The expression of PROS1-related genes such as GAS6, RhoA, FasL, Tyro-3, $A x l$, and Mertk was also quantified using qPCR and results were calculated based on the $\Delta \Delta \mathrm{Ct}$ method (26). ACTB gene was used as the reference gene. Primer sequences are shown in Table I.

Viability assay. The CellTiter-Glo ${ }^{\circledR}$ Luminescent Cell Viability Assay (Promega Corp.) which is based on the quantification of ATP present in the viable cells was used for viability assay. Cells were cultured for 24 and $48 \mathrm{~h}$ post-transfection. Subsequently, CellTiter-Glo ${ }^{\circledR}$ buffer was added onto the CellTiter-Glo ${ }^{\circledR}$ substrate, which was then loaded into the samples. The luminescent signal was captured at $570 \mathrm{~nm}$ using SpectraMax ${ }^{\circledR}$ L Luminescence Microplate Reader (Molecular Devices, LLC, Sunnyvale, CA, USA). Cellular viability was calculated based on the normalization between treated (siPROS1) vs. non-targeting siRNA cells from three independent experiments.

Proliferation assay. Proliferation assay was performed using the bromodeoxyuridine (BrdU) incorporation method (Millipore Corp., Billerica, MA, USA). Transfected cells were cultured for $24 \mathrm{~h}$ in the present of BrdU which was incorporated into newly synthesized DNA strand of the proliferating cells. The cells were then fixed, and incubated with anti-BrdU monoclonal antibody (Millipore Corp.) for $1 \mathrm{~h}$. Goat anti-mouse IgG peroxidase was added onto the well. Incorporation of BrdU in the proliferating cells leads to colorimetric changes from clear to blue which was measured using Varioskan Flash Multimode Reader (Thermo Fisher Scientific Oy, Vantaa, Finland) at $450 \mathrm{~nm}$ wavelength.

Migration assay. The effect of PROS1 gene silencing on tumor cell invasion was investigated using $\mathrm{QCM}^{\mathrm{TM}} 3 \mu \mathrm{m}$ 24-well Chemotaxis Cell Migration Assay kit (Millipore Corp.). Cells were seeded in the 24 -well inserts at a density of $1 \times 10^{4}$ 
Table I. Primer sequences used for qPCR analysis.

\begin{tabular}{|c|c|}
\hline Gene & Primer sequence $\left(5^{\prime} \rightarrow 3^{\prime}\right)$ \\
\hline \multirow[t]{2}{*}{ PROS1 } & F TGCTGGCGTGTCTCCTCCTA \\
\hline & R CAGTTCTTCGATGCATTCTCTTTCA \\
\hline \multirow[t]{2}{*}{ Tyro-3 } & F CACGGTAGAAGGTGTGCCAT \\
\hline & R TGGTAACAGGTTCAGGGGGA \\
\hline \multirow[t]{2}{*}{$A x l$} & F TTAGTGCTACGCGGAATGGG \\
\hline & R TGTCCATTAGCACCTCTGGG \\
\hline \multirow[t]{2}{*}{ Mertk } & F GTCCATCTGTCCATCCGTCC \\
\hline & R CCTCAGTGATAGCTCTACGCC \\
\hline \multirow[t]{2}{*}{ Gas6 } & F ACGACCCCGAGACGGATTA \\
\hline & R GCGAAGCCTGAGTTTTTGGT \\
\hline \multirow[t]{2}{*}{ FasL } & F CCTTGGTAGGATTGGGCCTG \\
\hline & R CTGTGTGCATCTGGCTGGTA \\
\hline
\end{tabular}

F, forward; R, reverse.

cells/well in serum-free media for $24 \mathrm{~h}$ and allowed to migrate through the membrane towards the media. The migrated cells were then lysed and the fluorescent signal was quantified using Varioskan Flash Multimode Reader (Thermo Fisher Scientific Oy). We also performed wound healing scratch assay in order to observe the cellular motility in siPROS1-treated LN18 cells. Wound closure was observed at 0, 24, 48 and $72 \mathrm{~h}$ post-scratching. These assays were performed in three independent replicates.

Invasion assay. The role of PROS1 in cell invasion was investigated using QCM ${ }^{\mathrm{TM}}$ 24-well Cell Invasion Assay kit (Millipore Corp.). The cells were cultured overnight in serum-free media and allowed to invade through the ECM. The cells were harvested and lysed prior to fluorometric quantification using Varioskan Flash Multimode Reader (Thermo Fisher Scientific, Inc.). The invasion assay was carried out in three independent replicates.

Apoptosis assay. Apoptosis was determined using the ssDNA Apoptosis ELISA kit (Millipore Corp.). In total, 5x10 32 LN18 cells were grown overnight in a 96-well plate. Subsequently, the cells were transfected either with siPROS1 or non-targeting siRNA for $48 \mathrm{~h}$. Cells were then prepared for apoptosis measurement according to the manufacturer's protocol and the signal was measured using ELx800 TC models 95 Microplate Reader (Biotek Instruments, Inc., Winooski, VT, USA).

Cell cycle analysis. Cell cycle assay was performed using $1 \times 10^{6}$ LN18 cells that were transfected with siPROS1 or non-targeting siRNA. Cells were harvested using a standard protocol as indicated in the Cycletest ${ }^{\mathrm{TM}}$ Plus DNA Reagent Kit protocol (BD Biosciences, Mississauga, ON, Canada). Subsequently, cells were washed three times with wash buffer. Cells were then suspended in solution A containing trypsin. Solution B with trypsin inhibitor and RNase were then added into the cell suspension. Finally, solution $\mathrm{C}$ which contained propidium iodide (PI) was added. Flow cytometric analysis was performed using BD FACSAria ${ }^{\mathrm{TM}}$ (BD Biosciences,
Franklin Lakes, NJ, USA). Data were analysed using ModFit LT software (Verity Software House, Inc., Topsham, ME, USA). The percentage of arrested cells was measured by the percentage of hypodiploid cells accumulated at the G0/G1, S, $\mathrm{G} 2 / \mathrm{M}$ checkpoints of the cell cycle.

Western blotting. Protein expression of PROS1 was assessed using western blotting. Cells were treated with siPROS1 and proteins were harvested and extracted using radioimmunoprecipitation assay (RIPA) buffer. A total of $50 \mu \mathrm{g}$ protein was loaded onto the Mini-PROTEAN ${ }^{\circledR}$ Precast Gels (Bio-Rad Laboratories, Inc.), and then transferred onto the Immobilon transfer membranes (Millipore Corp.). Membranes were then incubated with SuperBlock ${ }^{\circledR}$ (Thermo Fisher Scientific, Inc.) for $1 \mathrm{~h}$ at room temperature. After that, membranes were incubated overnight with PROS1 mouse monoclonal antibody (1:500; Santa Cruz Biotechnology, Inc., Dallas, TX, USA) at $4^{\circ} \mathrm{C}$. The membranes were then washed three times with TBST. Membranes were then incubated with goat anti-mouse secondary antibody conjugated to alkaline phosphate $(1: 2,000$; Santa Cruz Biotechnology, Inc.) for $1 \mathrm{~h}$ at room temperature. Prior to protein detection, the membranes were washed three times. Finally, proteins were detected using Pierce ECL and SuperSignal substrate (Thermo Fisher Scientific, Inc.). $\beta$-actin was used as an internal control.

Protein array. Protein array was conducted using the Human Apoptosis Array kit (RayBiotech, Norcross, GA, USA). Protein samples were extracted from $48 \mathrm{~h}$ post-transfection according to the manufacturer's protocol. The quantity of the protein isolated was determined using BCA Protein Assay (Thermo Fisher Scientific, Inc.). Briefly, protein was loaded into the chamber slides coated with 43 different types of apoptosis antibodies. Subsequently, the slides were washed and the membranes were incubated with a cocktail of biotin-conjugated anti-apoptotic protein antibodies. The membranes were incubated with HRP-streptavidin prior to signal detection.

Statistical analysis. All data were expressed as the mean \pm SD of three independent experiments. Significant differences were defined as $\mathrm{p}<0.05$. All statistical analyses were performed using Microsoft Excel and the Statistical Package for the Social Sciences (SPSS) software.

\section{Results}

PROS1 as a novel candidate for GBM therapy. Meta-analysis on five microarray datasets (Bredel Brain 2, Lee Brain, Liang Brain, Shai Brain, and Sun Brain) identified 460 upregulated genes based on the median rank and $p<0.05$. All datasets were normalized between cancers vs. normal tissues. These 460 genes were used as candidates for RNAi screening. Based on the SSMD and kMAD analyses, 212 hits were identified. After selection, PROS1 was identified as a target gene for validation since the role of PROS1 in GBM has not been documented (Fig. 1).

PROS1 silencing decreases PROS1 mRNA and protein expressions. The efficiency of PROS1 silencing was assessed using qPCR. The results showed that $\sim 80 \%$ of the PROS1 gene was 
A

\begin{tabular}{|c|c|c|c|c|c|c|}
\hline & Gene & 1 & 2 & 3 & 4 & 5 \\
\hline & PROS1 & & & & & \\
\hline $\begin{array}{l}1 . \\
2 . \\
3 . \\
4 . \\
5 .\end{array}$ & $\begin{array}{l}\text { Bredel Brain } 2 \\
\text { Lee Brain, Ca } \\
\text { Liang Brain, } P \\
\text { Shai Brain, On } \\
\text { Sun Brain, Ca }\end{array}$ & $\begin{array}{l}\text { Res, } \\
2006 \\
\text { cad } \\
003 \\
2006\end{array}$ & USA & & & \\
\hline
\end{tabular}

C

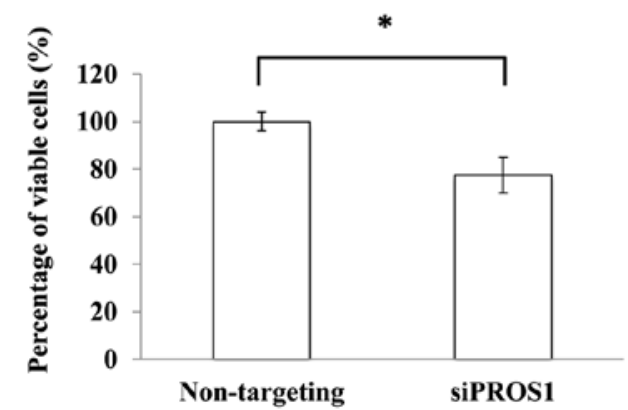

B

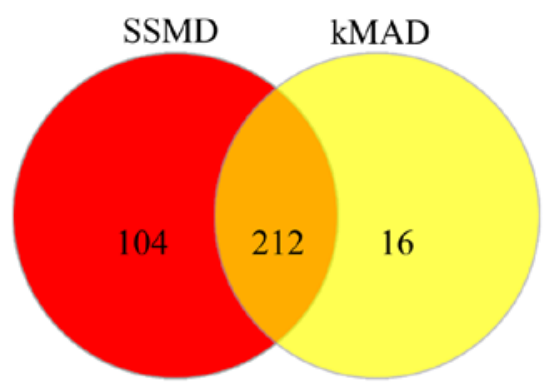

D

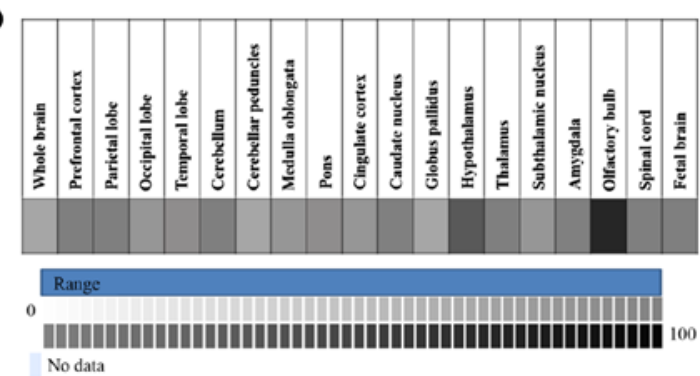

Figure 1. Meta-analysis and RNAi screening identified PROS1 as a novel candidate for GBM therapy. (A) Meta-analysis of PROS1 expression on five microarray datasets from Oncomine database. (B) A total of 212 hits overlapped by using SSMD and kMAD methods. (C) High-throughput RNAi screening shows that PROS1 gene silencing significantly reduced cell viability in GBM cells. ${ }^{*} \mathrm{P}<0.05$. (D) The expression of PROS1 in different parts of the brain. RNAi, RNA interference; GBM, glioblastoma multiforme.

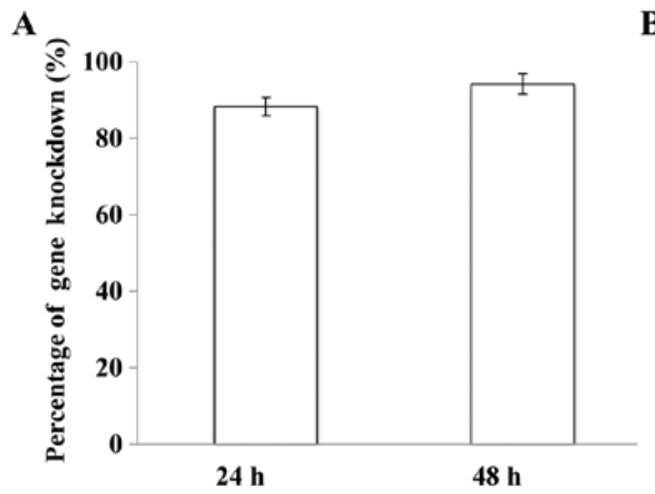

B

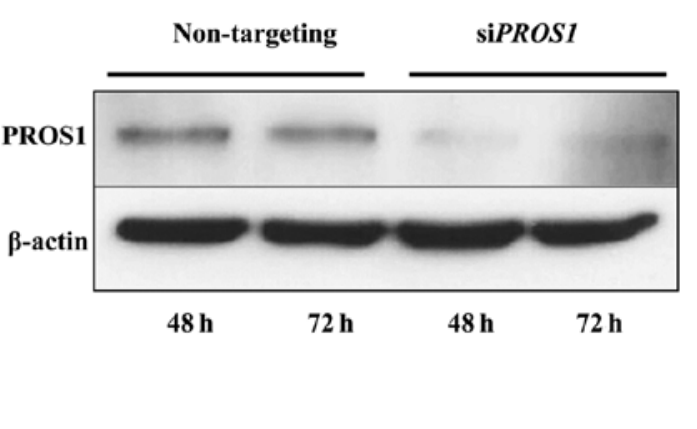

Figure 2. PROS1 gene silencing results in reduced PROS1 protein expression. (A) The efficiency of PROS1 gene knockdown was $80 \%$ at $24 \mathrm{~h}$ and increased up to $\sim 100 \%$ at $48 \mathrm{~h}$. (B) Western blotting of PROS1 was performed to determine the knockdown efficiency at protein level. The results showed that the PROS1 protein expression was depleted $48 \mathrm{~h}$ post-transfection.

knocked down after $24 \mathrm{~h}$ and it increased up to $100 \%$ after $48 \mathrm{~h}$ of transfection. This was further confirmed at protein level via western blotting at 48 and $72 \mathrm{~h}$ post-silencing as the expression of the PROS1 protein was reduced significantly compared to control (Fig. 2).

PROS1 silencing reduces cell viability and cell proliferation. Cell viability assay was conducted using CellTiter-Glo ${ }^{\circledR}$ Luminescent Cell Viability Assay to determine the effect of PROS1 gene silencing. The number of viable cells was reduced in a time-dependent manner. The quantification of proliferating cells by BrdU showed that the proliferation signal was decreased in siPROSI treatment (18\%) compared to the control (Fig. 3).

PROS1 silencing inhibits LN18 cell migration. Migration was reduced significantly $(\mathrm{p}<0.05)$ in siPROSI-treated LN18 cells.
The scratch assay demonstrated the inhibition of the migratory potential of the 24-h post-scratch siPROS1-treated LN18 cells. Surprisingly, the size of wound scratch remained up to $72 \mathrm{~h}$. These data suggest the possible role of PROS1 in GBM cell migration (Fig. 4A and B).

PROS1 silencing reduces GBM cell invasion. Cell invasion assay was performed to study whether PROS1 suppression could influence the invasion of LN18 cells. The results showed that the invasion of LN18 cells through the ECM was inhibited with siPROSl-treated cells up to $82 \%(\mathrm{p}<0.01)$ compared to the control group (Fig. 4C).

PROS1 silencing significantly induces cell death through apoptosis. ELISA-based assay was conducted to determine the mode of cell death in siPROS1-treated LN18 cells. Apoptosis was increased compared to the control at $48 \mathrm{~h}$ post-transfection 
A

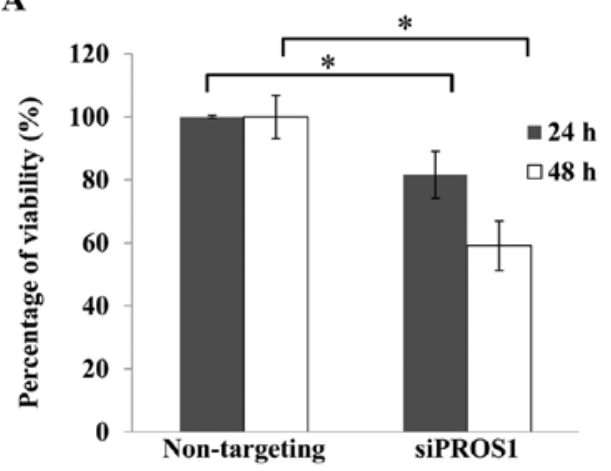

B

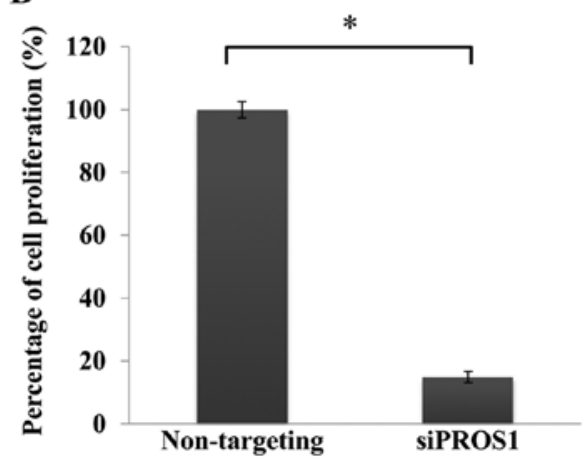

Figure 3. PROS1 gene silencing significantly reduces viability and cell proliferation. (A) PROS1 gene silencing resulting in reduced GBM cell viability up to $40 \%$ at $48 \mathrm{~h}$ post-transfection. ${ }^{\mathrm{P}}<0.05$. (B) Treatment of GBM cells with siPROS1 showed decreased cell proliferation at $48 \mathrm{~h}$ post-transfection by BrdU assay. GBM, glioblastoma multiforme; BrdU, bromodeoxyuridine.

A

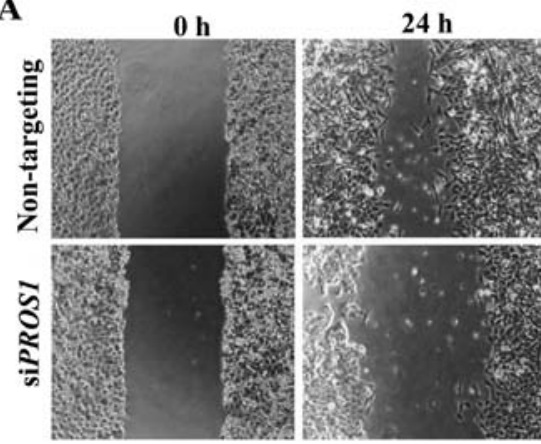

B

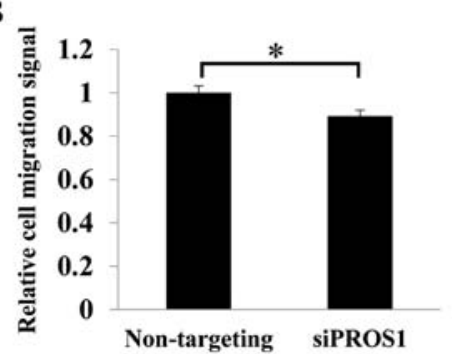

D

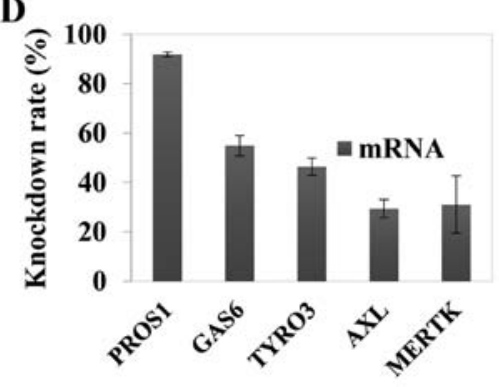

$48 \mathrm{~h}$

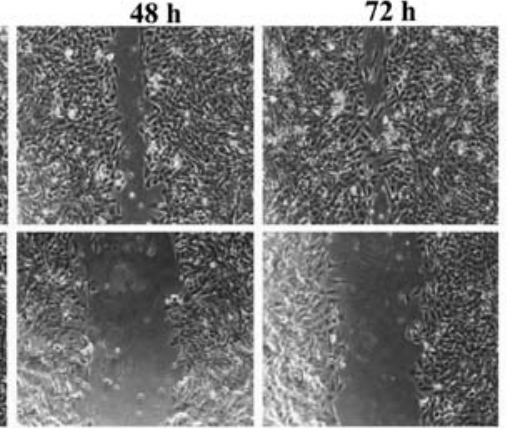

C

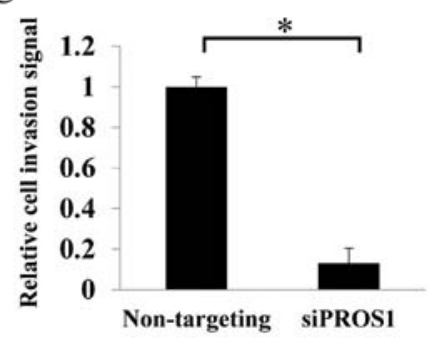

$\mathbf{E}$

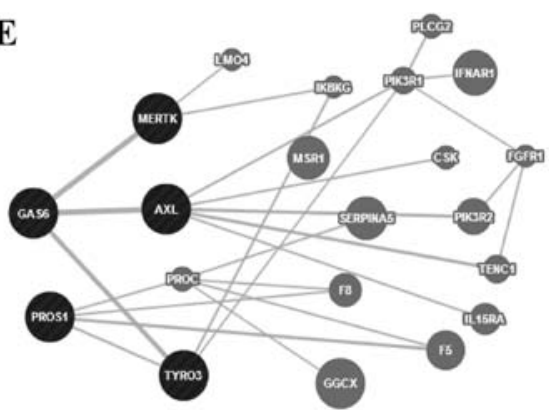

Figure 4. PROS1 silencing in GBM cells exhibits decreased migratory potential and may become a potential target for cell invasion. Silencing of PROS1 gene showed a significant reduction in cell migration and invasion in GBM cells at 48 h post-transfection. (A) The morphological observation of $P R O S 1$ gene silencing effects on wound healing assay in GBM cells shows a clear gating in siPROS1 treatment scratched cells at 0-72 h compared to control. (B) PROS1 gene silencing significantly reduces migratory potential through membrane in GBM cells. (C) PROS1 gene silencing significantly controls cancer invasiveness by reducing the invasion capability in GBM cells. ${ }^{*} \mathrm{P}<0.05$. (D) PROS1 gene silencing reduced the mRNA expression of Gas6 and TAM receptors. (E) Mapping of physical interaction between PROS1, GAS6 and TAM receptors was generated by GeneMANIA. GBM, glioblastoma multiforme.

$(\mathrm{p}<0.05)$ (Fig. 5A). There was no evidence of cell cycle arrests identified from the cell cycle assay (data not shown). Further validation was conducted using protein array to elucidate the relevant pathways involved in this process.
PROS1 gene silencing leads to decreased expression of Tyro-3, Axl and Mertk. qPCR was performed to study the effect of PROS1 silencing on its related interacting genes from the TAM family of receptor tyrosine kinases which include 
A

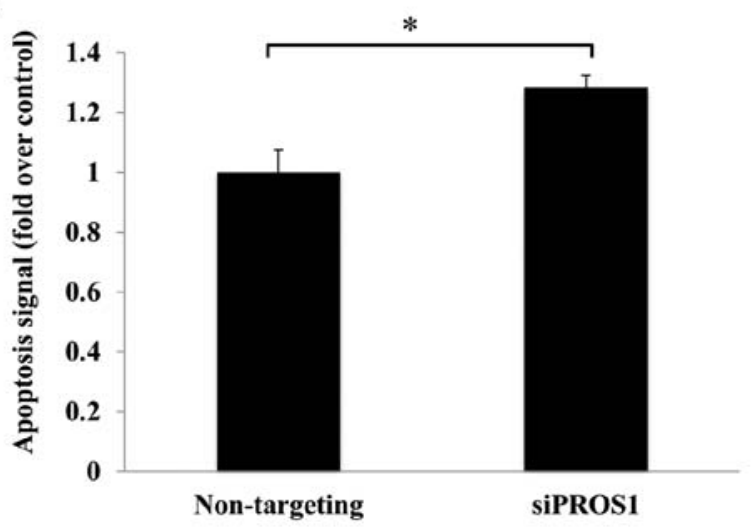

C

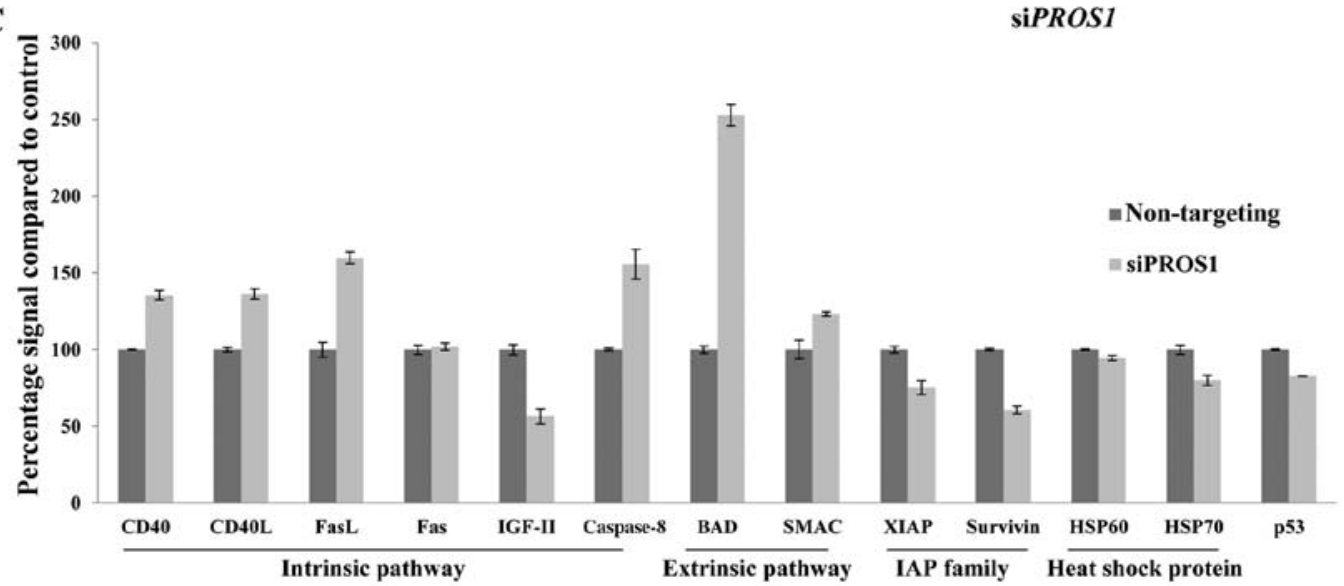

B

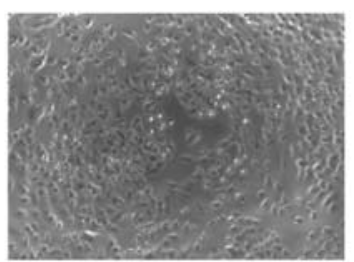

Non-targeting

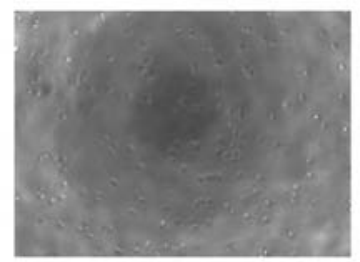

siPROS1

Figure 5. The PROS1 gene silencing significantly induces cell death through apoptosis by activating both intrinsic and extrinsic pathways. (A) Silencing of PROS1 resulted in an apoptosis signal at $48 \mathrm{~h}$ post-transfection. ${ }^{*} \mathrm{P}<0.05$. (B) siPROS1 treatment induced formation of apoptotic bodies. (C) Most significant hits induced by PROS1 gene silencing in apoptosis array indicate the activation of both intrinsic and extrinsic pathways.

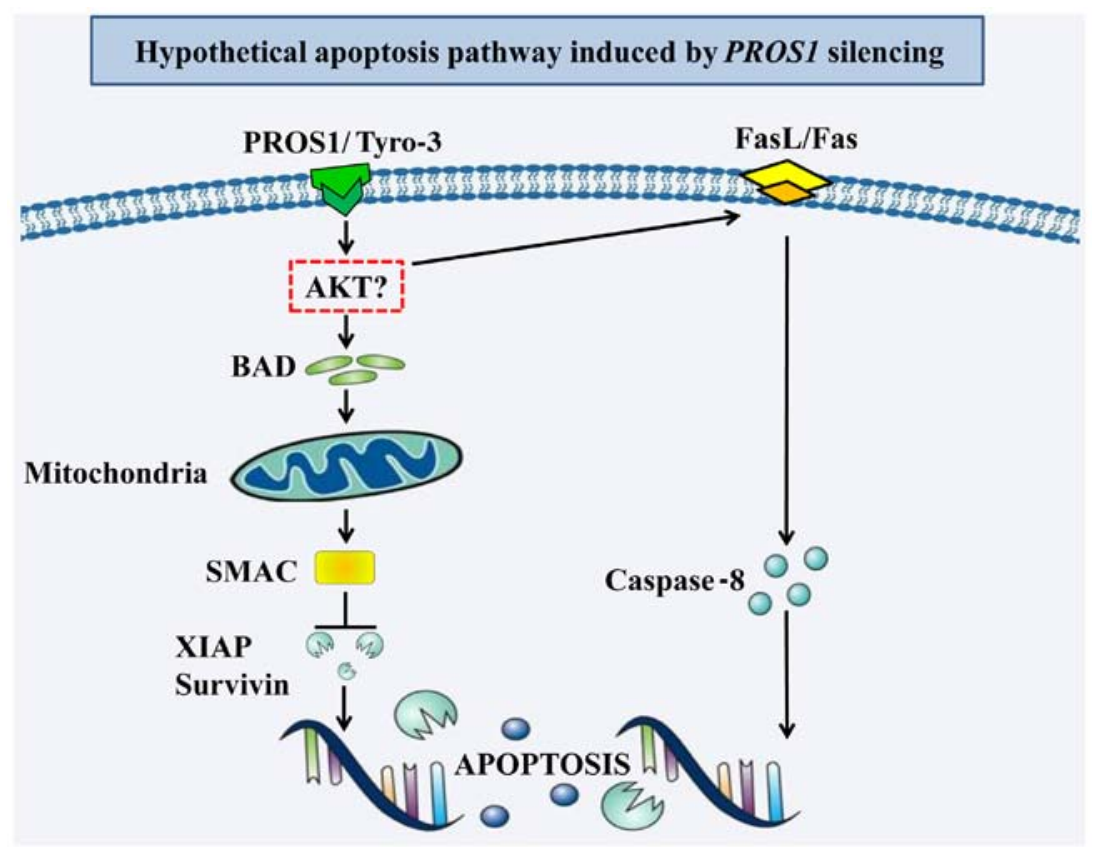

Figure 6. The working model of intrinsic and extrinsic pathways induced by PROS1 gene silencing. In the intrinsic pathway, PROS1 silencing activates BAD which is a BCL2-associated antagonist of cell death and then activates the mitochondrial pathway by releasing SMAC protein. The overexpression of SMAC will suppress the expression of IAP family which consists of XIAP and survivin and then induce apoptosis via downstream of apoptosis caspases. In the extrinsic pathway, an overexpression of FasL/Fas activated caspase-8 and induced apoptosis via downstream of apoptosis caspases. In the presence of PROS1, AKT will be activated and then phosphorylated BAD protein. The activation of AKT also suppresses the expression of FasL/Fas and then blocks the caspase- 8 activation. Previously it was shown that the activation of AKT promotes overexpression of apoptosis inhibitors like BCL2 and IAP family and also suppresses the expression of pro-apoptotic proteins like BAD and BAX. IAP, inhibitor of apoptosis protein; FasL, Fas ligand. 
GAS6, Tyro-3, Axl, and Mertk. This was performed at $48 \mathrm{~h}$ post-transfection. The level of GAS6 was reduced to $68.7 \%$ compared to the control $(\mathrm{p}<0.05)$. PROS1 gene silencing also reduced the expression of the tyrosine kinases, especially the Tyro-3 where the expression was 50\% reduced compared to the control. The expression of $A x l$ and Mertk genes was reduced to 70.6 and $69 \%$, respectively (Fig. 4D and 4E).

\section{Discussion}

The main aim of this study was to understand the functional role of PROS1 in GBM by performing silencing experiments coupled with various functional assays. PROS1 was identified as a potential gene target for GBM from our meta-analysis using five microarray datasets and the loss-of-function RNAi screening of 460 upregulated genes. PROS1 is a vitamin K-dependent plasma protein and is known to be involved in the anticoagulant cascade. It acts as a cofactor for anticoagulant protease in the blood coagulation system known as the activated protein C (APC) (27). PROS1 shares $\sim 43 \%$ of amino acid identity with GAS6, a $\gamma$-carboxyglutamic acid (Gla)-containing protein, which stimulates cell proliferation through activation of the Axl receptor tyrosine kinase (28-30). PROS1 and GAS6 are ligands for Axl together with Tyro-3 and Mertk and were reported to be overexpressed in haematological malignancies and solid tumors, suggesting that these molecules activate important autocrine-based oncogenic signaling events in cancer cells (31-34). Overexpression of TAM receptors mediates multiple oncogenic phenotypes in GBM such as in vitro proliferation, anchorage-independent growth, xenograft growth, resistance to apoptosis, autophagy, invasion and migration as well as activation of the downstream PI3K and MAPK survival pathways (33). Inhibition of Mertk and Axl by gene knockdown in astrocytoma cells enhanced apoptosis and improved chemosensitivity towards conventional chemotherapeutic agents such as temozolomide, carboplatin and vincristine (35).

Initially PROS1 was thought to be the ligand for the TAM receptors. However, Stitt et al have shown that PROS1 has a higher affinity for the Tyro-3 receptor and can transform NIH $3 \mathrm{~T} 3$ cells in an autocrine manner (36). One of the important findings that changed the perspective for PROS1 was that the anticoagulant factor played an important role in activating Tyro-3 activity as its expression was upregulated in cultured Schwann cells and astrocytes following nerve injury (36). This activation of intracellular signaling cascades by specific cell-surface receptors would promote cell proliferation for tissue repair and growth. PROS1 was found to be highly expressed in high-grade prostate cancers suggesting that it has an important role in the regulation of cancer cell survival (29). Knockdown of PROSI by shRNA was reported to significantly reduce the number of cancerous cells in a time-dependent manner (37). Indeed, this is in agreement with our findings where silencing of PROS1 using siRNA significantly reduced cell viability of GBM cells by $>40 \%$. This was also supported by the reduction of Brdu proliferative signals.

PROS1 is also involved in the phagocytosis of apoptotic cells in the immune, nervous, and reproductive systems through interaction with Tyro-3 (38). During hypoxia or ischemia, PROS1 protects neuron cells and inhibits apoptosis by inhibiting Fas ligand (FasL) production and inhibiting FasL-dependent caspase- 8 activation within the extrinsic apoptotic pathway (39). Wang et al showed that Tyro-3 silencing affected several important signaling pathways including P13K/AKT, Wnt/ $\beta$-catenin, ERK/MAPK, PAK/JNK, JAK/Stat and $T G F-\beta$ as well as the retinoic acid receptor $(R A R)$ activation (40). We showed that silencing PROS1 led to a significant increase in apoptotic signals and this result was validated using protein array. Silencing of PROS1 caused significant activation of the apoptotic pathways by upregulation of CD40, CD40L, Fas, FasL and caspase- 8 of the intrinsic pathway as well as BAD and SMAC of the extrinsic pathway. Interestingly, it significantly reduced the expression of the inhibitor of apoptosis protein (IAP), XIAP and survivin. PROS1 silencing also led to the downregulation of Tyro-3, Axl, Mertk and Gas6 gene expressions, suggesting that the GBM cells might undergo apoptosis through the TAM receptor interaction. However, there are some limitations in terms of the number of protein markers available in our protein array which hindered the identification of other downstream apoptosis proteins involved.

Another study on castration-resistant prostate cancer cells showed that the addition of human purified PROS1 increased the migration of these cells (29). Furthermore, the high-throughput wound healing screening on the epithelial cells revealed the involvement of TAM receptors in cell migration (41). Our results are consistent as we showed that PROS1 gene silencing delayed the wound enclosure in GBM cells and significantly reduced the capability of cells to migrate. This might be due to the involvement of the extracellular domains of TAM receptors that contain adhesion molecule-like motifs which controls cell-cell contacts and actin cytoskeleton regulation (42). Our results showed that silencing of PROS1 expression also led to a significant reduction in cell invasion through the ECM in GBM cells. These findings suggest that PROS1 may provide a survival advantage for advanced stage cancer like prostate and GBM by controlling cancer cell migration and invasion.

In summary, we showed that silencing PROS1 reduces survival, migration and invasion of GBM cells (as detailed in Fig. 6). It also activates apoptosis in GBM cells by activating the intrinsic and extrinsic apoptotic pathways. Further validation using in vivo studies are needed to enhance our understanding on the mechanistic role of PROS1 in GBM cells. This will hopefully allow the development of PROS1 gene therapy as a possible approach to increase patient survival and improve the treatment of GBM patients.

\section{Acknowledgements}

We would like to thank the Ministry of Education, Malaysia for the funding. This study was funded by the Higher Institution Centre of Excellence (HICoE) (grant no. JJ-008-2011), Ministry of Education, Malaysia.

\section{References}

1. Omuro A and DeAngelis LM: Glioblastoma and other malignant gliomas: A clinical review. JAMA 310: 1842-1850, 2013.

2. Davies AM, Weinberg U and Palti Y: Tumor treating fields: A new frontier in cancer therapy. Ann N Y Acad Sci 1291: 86-95, 2013. 
3. Parpura V, Heneka MT, Montana V, Oliet SHR, Schousboe A, Haydon PG, Stout RF Jr, Spray DC, Reichenbach A, Pannicke T, et al: Glial cells in (patho)physiology. J Neurochem 121: 4-27, 2012.

4. Huse JT, Holland E and DeAngelis LM: Glioblastoma: Molecular analysis and clinical implications. Annu Rev Med 64: 59-70, 2013.

5. Kanu OO, Hughes B, Di C, Lin N, Fu J, Bigner DD, Yan H and Adamson C: Glioblastoma multiforme oncogenomics and signaling pathways. Clin Med Oncol 3: 39-52, 2009.

6. Reardon DA, Conrad CA, Cloughesy T, Prados MD Friedman HS, Aldape KD, Mischel P, Xia J, DiLea C, Huang J, et al: Phase I study of AEE788, a novel multitarget inhibitor of ErbB- and VEGF-receptor-family tyrosine kinases, in recurrent glioblastoma patients. Cancer Chemother Pharmacol 69: $1507-1518,2012$.

7. Vitucci M, Karpinich NO, Bash RE, Werneke AM, Schmid RS, White KK, McNeill RS, Huff B, Wang S, Van Dyke T, et al Cooperativity between MAPK and PI3K signaling activation is required for glioblastoma pathogenesis. Neuro Oncol 15: $1317-1329,2013$.

8. Akhavan D, Cloughesy TF and Mischel PS: mTOR signaling in glioblastoma: Lessons learned from bench to bedside. Neuro-oncol 12: 882-889, 2010.

9. Nakada M, Kita D, Watanabe T, Hayashi Y, Teng L, Pyko IV and Hamada J: Aberrant signaling pathways in glioma. Cancers (Basel) 3: 3242-3278, 2011.

10. Ohgaki $\mathrm{H}$ and Kleihues P: Genetic pathways to primary and secondary glioblastoma. Am J Pathol 170: 1445-1453, 2007.

11. Zhang C, Moore LM, Li X, Yung WKA and Zhang W: IDH1/2 mutations target a key hallmark of cancer by deregulating cellular metabolism in glioma. Neuro Oncol 15: 1114-1126, 2013.

12. Pierscianek D, Kim YH, Motomura K, Mittelbronn M, Paulus W, Brokinkel B, Keyvani K, Wrede K, Nakazato Y, Tanaka Y, et al: MET gain in diffuse astrocytomas is associated with poorer outcome. Brain Pathol 23: 13-18, 2013.

13. Eisele G and Weller M: Targeting apoptosis pathways in glioblastoma. Cancer Lett 332: 335-345, 2013.

14. Krakstad C and Chekenya M: Survival signalling and apoptosis resistance in glioblastomas: Opportunities for targeted therapeutics. Mol Cancer 9: 135, 2010.

15. Panner A, Crane CA, Weng C, Feletti A, Parsa AT and Pieper RO A novel PTEN-dependent link to ubiquitination controls FLIPS stability and TRAIL sensitivity in glioblastoma multiforme. Cancer Res 69: 7911-7916, 2009.

16. Ruano Y, Mollejo M, Camacho FI, Rodríguez de Lope A, Fiaño C, Ribalta T, Martínez P, Hernández-Moneo JL and Meléndez B: Identification of survival-related genes of the phosphatidylinositol 3'-kinase signaling pathway in glioblastoma multiforme. Cancer 112: 1575-1584, 2008.

17. Guvenc H, Pavlyukov MS, Joshi K, Kurt H, BanasavadiSiddegowda YK, Mao P, Hong C, Yamada R, Kwon CH Bhasin D, et al: Impairment of glioma stem cell survival and growth by a novel inhibitor for Survivin-Ran protein complex. Clin Cancer Res 19: 631-642, 2013.

18. Catuogno S, Esposito CL, Quintavalle C, Condorelli G, de Franciscis V and Cerchia L: Nucleic acids in human glioma treatment: Innovative approaches and recent results. J Signal Transduct 2012: 735135, 2012.

19. Jensen SA, Day ES, Ko CH, Hurley LA, Luciano JP, Kouri FM, Merkel TJ, Luthi AJ, Patel PC, Cutler JI, et al: Spherical nucleic acid nanoparticle conjugates as an RNAi-based therapy for glioblastoma. Sci Transl Med 5: 209ra152, 2013.

20. Zhang Y, Zhang YF, Bryant J, Charles A, Boado RJ and Pardridge WM: Intravenous RNA interference gene therapy targeting the human epidermal growth factor receptor prolongs survival in intracranial brain cancer. Clin Cancer Res 10 : 3667-3677, 2004.

21. Wang XL, Xu R, Wu X, Gillespie D, Jensen R and Lu ZR: Targeted systemic delivery of a therapeutic siRNA with a multifunctional carrier controls tumor proliferation in mice. Mol Pharm 6: 738-746, 2009.

22. Bumcrot D, Manoharan M, Koteliansky V and Sah DWY: RNAi therapeutics: A potential new class of pharmaceutical drugs. Nat Chem Biol 2: 711-719, 2006.

23. Rolle K, Nowak S, Wyszko E, Nowak M, Zukiel R, Piestrzeniewicz R, Gawronska I, Barciszewska MZ and Barciszewski J: Promising human brain tumors therapy with interference RNA intervention (iRNAi). Cancer Biol Ther 9 : $396-406,2010$
24. Zukiel R, Nowak S, Wyszko E, Rolle K, Gawronska I, Barciszewska MZ and Barciszewski J: Suppression of human brain tumor with interference RNA specific for tenascin-C. Cancer Biol Ther 5: 1002-1007, 2006.

25. Rhodes DR, Yu J, Shanker K, Deshpande N, Varambally R, Ghosh D, Barrette T, Pandey A and Chinnaiyan AM: ONCOMINE: A cancer microarray database and integrated data-mining platform. Neoplasia 6: 1-6, 2004.

26. Livak KJ and Schmittgen TD: Analysis of relative gene expression data using real-time quantitative PCR and the 2(-Delta Delta C(T)) method. Methods 25: 402-408, 2001.

27. Dahlbäck B: The tale of protein $\mathrm{S}$ and $\mathrm{C} 4 \mathrm{~b}$-binding protein, a story of affection. Thromb Haemost 98: 90-96, 2007.

28. Hafizi S and Dahlbäck B: Gas6 and protein S. Vitamin $\mathrm{K}$-dependent ligands for the Axl receptor tyrosine kinase subfamily. FEBS J 273: 5231-5244, 2006.

29. Saraon P, Musrap N, Cretu D, Karagiannis GS, Batruch I, Smith C, Drabovich AP, Trudel D, van der Kwast T, Morrissey C, et al: Proteomic profiling of androgen-independent prostate cancer cell lines reveals a role for protein $\mathrm{S}$ during the development of high grade and castration-resistant prostate cancer. J Biol Chem 287: 34019-34031, 2012.

30. Suleiman L, Négrier C and Boukerche H: Protein S: A multifunctional anticoagulant vitamin K-dependent protein at the crossroads of coagulation, inflammation, angiogenesis, and cancer. Crit Rev Oncol Hematol 88: 637-654, 2013.

31. Lemke G: Biology of the TAM receptors. Cold Spring Harb Perspect Biol 5: a009076, 2013

32. Lemke $G$ and Rothlin CV: Immunobiology of the TAM receptors. Nat Rev Immunol 8: 327-336, 2008.

33. Linger RMA, Keating AK, Earp HS and Graham DK: Taking aim at Mer and Axl receptor tyrosine kinases as novel therapeutic targets in solid tumors. Expert Opin Ther Targets 14: 1073-1090, 2010.

34. Wimmel A, Rohner I, Ramaswamy A, Heidtmann HH, Seitz R, Kraus $M$ and Schuermann M: Synthesis and secretion of the anticoagulant protein $\mathrm{S}$ and coexpression of the Tyro3 receptor in human lung carcinoma cells. Cancer 86: 43-49, 1999.

35. Keating AK, Kim GK, Jones AE, Donson AM, Ware K, Mulcahy JM, Salzberg DB, Foreman NK, Liang X, Thorburn A, et al: Inhibition of Mer and Axl receptor tyrosine kinases in astrocytoma cells leads to increased apoptosis and improved chemosensitivity. Mol Cancer Ther 9: 1298-1307, 2010.

36. Stitt TN, Conn G, Gore M, Lai C, Bruno J, Radziejewski C, Mattsson K, Fisher J, Gies DR, Jones PF, et al: The anticoagulation factor protein $\mathrm{S}$ and its relative, Gas6, are ligands for the Tyro 3/Axl family of receptor tyrosine kinases. Cell 80: 661-670, 1995.

37. Saraon P, Jarvi K and Diamandis EP: High-throughput proteomic analysis identifies protein $\mathrm{s}$ as a modulator of high grade and castrate-resistant prostate cancer. Cancer Res (AACR Annual Meeting abstracts) 72 (8 Suppl): LB-293, 2012.

38. Lemke $\mathrm{G}$ and Burstyn-Cohen T: TAM receptors and the clearance of apoptotic cells. Ann N Y Acad Sci 1209: 23-29, 2010.

39. Guo H, Barrett TM, Zhong Z, Fernández JA, Griffin JH, Freeman RS and Zlokovic BV: Protein S blocks the extrinsic apoptotic cascade in tissue plasminogen activator/N-methyl D-aspartate-treated neurons via Tyro3-Akt-FKHRL1 signaling pathway. Mol Neurodegener 6: 13, 2011

40. Wang Y, Moncayo G, Morin P Jr, Xue G, Grzmil M, Lino MM, Clément-Schatlo V, Frank S, Merlo A and Hemmings BA: Mer receptor tyrosine kinase promotes invasion and survival in glioblastoma multiforme. Oncogene 32: 872-882, 2013.

41. Simpson KJ, Selfors LM, Bui J, Reynolds A, Leake D, Khvorova A and Brugge JS: Identification of genes that regulate epithelial cell migration using an siRNA screening approach. Nat Cell Biol 10: 1027-1038, 2008.

42. Vajkoczy P, Knyazev P, Kunkel A, Capelle HH, Behrndt S, von Tengg-Kobligk H, Kiessling F, Eichelsbacher U, Essig M, Read TA, et al: Dominant-negative inhibition of the Axl receptor tyrosine kinase suppresses brain tumor cell growth and invasion and prolongs survival. Proc Natl Acad Sci USA 103: 5799-5804, 2006. 